\title{
Efficacy and Safety of Intralesional Bleomycin in the Management of Ungual Warts
}

\author{
Archana Singal Chander Grover \\ Department of Dermatology and STD, University College of Medical Sciences and G.T.B. Hospital, University of \\ Delhi, New Delhi, India
}

\author{
Keywords \\ Periungual warts · Subungual warts · Intralesional · \\ Bleomycin
}

\begin{abstract}
Introduction: Ungual warts are common fibroepithelial tumours of the nail unit caused by human papillomavirus. Although various treatment modalities are available, their management is challenging due to high potential for recurrence and treatment-related nail dystrophy. Objective: This study evaluates the efficacy and safety of intralesional bleomycin injections in the treatment of ungual warts. Methods: Medical records of 80 patients with ungual warts, treated by us from 2015 to 2019, were analysed retrospectively with respect to the number of warts, site, morphology, and treatment outcome. Children $<5$ years of age, and pregnant and lactating women were excluded. All ungual warts were treated with intralesional injections of bleomycin 3 Units $/ \mathrm{mL}$, given at 3-4 weeks interval. Results and Conclusions: $A$ total of 250 periungual and subungual warts were treated in 80 patients. An average of 2.61 sessions per patient was required for complete resolution. Moderate pain was reported by 65 patients (81.2\%), and transitory perilesional hyperpigmentation was observed in 155 lesions (62\%). Severe pain and necrosis developed in 3 lesions (1.2\%) that were reversible
\end{abstract}

without any residual scarring and deformity. We conclude that intralesional bleomycin is an effective and safe therapeutic modality for ungual warts.

(c) 2020 S. Karger AG, Base

\section{Introduction}

Periungual and subungual warts are the most common fibroepithelial, benign tumours of the nail apparatus caused mostly by the human papillomavirus serotypes 1 , 2, 4, and 27 [1]. Lesions are weakly contagious, and their morphologic characteristics depend on their localization. The lesions are round like those on other body sites, when present on the proximal nail fold; oval and fissured over the lateral nail folds; and have a slightly hyperkeratotic rim in the hyponychium. Subungual warts often raise the nail plate and may even lead to nail plate damage. Although their natural life span is around 2-5 years with a tendency to spontaneous resolution, it is advisable to treat ungual warts to prevent spread to other body sites and other people [2].

Although the diagnosis of ungual warts is mainly clinical, a confirmation based on biopsy or dermoscopy is desirable for long-standing refractory lesions in elderly patients, in order to differentiate from clinical mimics like squamous

$\begin{aligned} & \text { karger@karger.com } \\ & \text { www.karger.com/sad }\end{aligned}$
Karger ${ }^{\prime /}$

Archana Singal

Department of Dermatology and STD University College of Medical Sciences and G.T.B. Hospital University of Delhi

Street-1, Delhi 110095 (India)

archanasingal@hotmail.com 
Fig. 1. a Periungual wart before treatment. b Haemorrhagic and necrotic crust, 5 days after the 1st intralesional bleomycin (3 U/ $\mathrm{mL})$. c Complete resolution of the lesion after 2 injections.
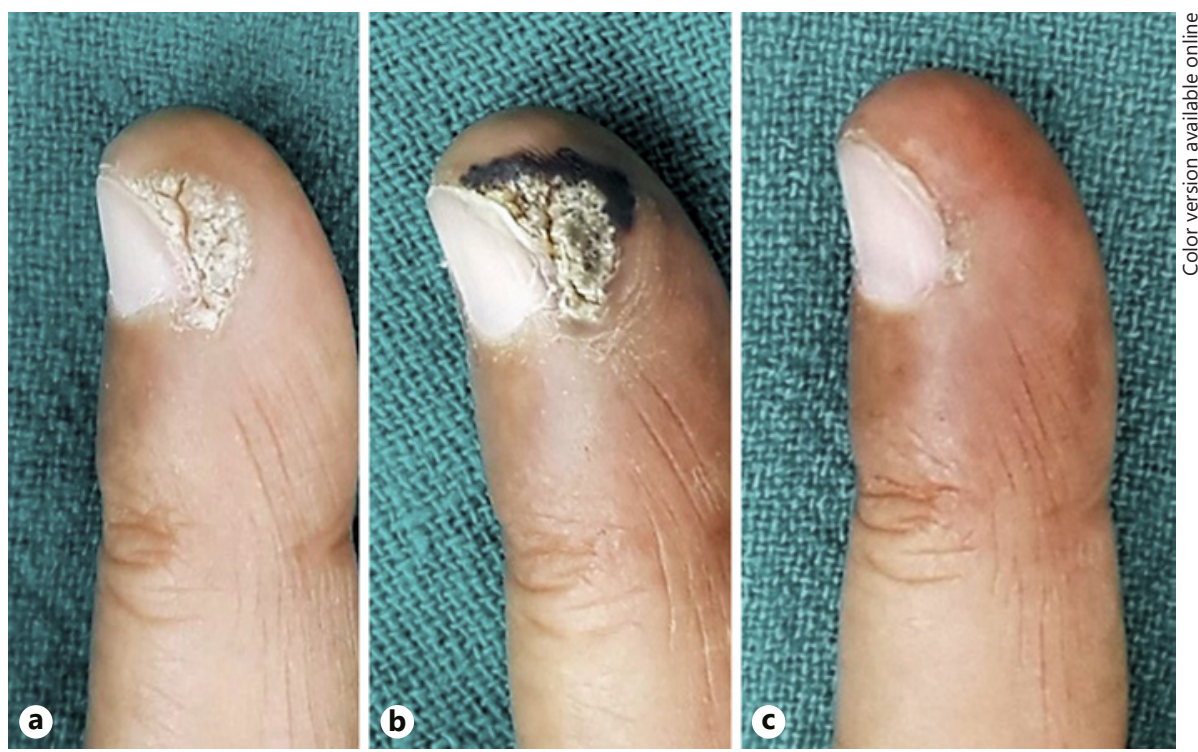

cell carcinoma or Bowen's disease. Despite the availability of numerous therapeutic options like radiofrequency ablation, chemical cautery, electrocautery, cryosurgery, surgical excision, and device-based therapies, treatment of ungual warts remains challenging because of variable outcome, high recurrence rate, and significant potential for nail dystrophy. The use of systemic drugs, immunotherapy, or chemotherapy may not be justifiable for localized lesions as these treatments are not targeted, suffer from poor penetration at the site of lesion, and can have systemic side effects.

Bleomycin is a cytotoxic agent that inhibits DNA and protein synthesis and has been used in the treatment of viral warts for $>5$ decades [3]. Many recent reports have shown encouraging results with intralesional bleomycin in ungual warts [4]. Two techniques have been used: multi-puncture technique and intralesional injection of bleomycin. For the latter, varying concentrations of bleomycin ranging from 1 to $3 \mathrm{U} / \mathrm{mL}$ has been used. In this retrospective study, we evaluated the efficacy and safety of intralesional bleomycin in a concentration of $3 \mathrm{U} / \mathrm{mL}$ given at 3-4 weeks interval.

\section{Materials and Methods}

\section{Patient Recruitment}

Meticulous records of patients treated for ungual warts in the Dermatology Department of University College of Medical Sciences \& GTB Hospital, New Delhi, India, from 2015 to 2019 were analysed. The research was conducted in accordance with the guidelines for human studies as per the World Medical Association Declaration of Helsinki. The diagnosis of periungual or subungual warts had been made clinically and confirmed on dermos- copy in all cases and/or by histopathology in selected cases where squamous cell carcinoma and Bowen's were considered in the differential diagnosis. Children below 5 years of age; elderly people with severe systemic disease, peripheral vascular disease, and connective tissue disease; heavy smokers; pregnant and lactating mothers; and patients with existing nail dystrophy secondary to other inflammatory or infective nail disorders were excluded from the study. Written informed consent was obtained from all eligible patients after explaining the painful nature of the injection, other treatment options available, and need for multiple visits and at least 3 months of follow-up after the last injection. Sequentially maintained photographs of the treated lesions were analysed.

\section{Preparation of Injection and Technique of Injection}

For injection, we used a stock solution of 15 Units of bleomycin powder, reconstituted in $5 \mathrm{~mL}$ of physiologic saline solution so as make a concentration of $3 \mathrm{U} / \mathrm{mL}$. This stock solution was used within maximum 60 days and stored at 4-6 degree temperature. Before injection, the solution was brought to room temperature and loaded in a 1-mL insulin syringe with a 30 -gauze pre-attached needle. Hyperkeratotic lesions were scraped with a surgical blade before the injection. Approximately $0.1-0.3 \mathrm{~mL}$ was injected per lesion. The needle was withdrawn once the lesion blanched or a tiny drop of the solution became visible on the surface of the lesion. Patients were counselled about the formation of an eschar or haemorrhagic crust over the next 3-5 days that tends to fall off in about 1-2 weeks' time. Application of generous amount of emollient (white soft paraffin and liquid paraffin cream) over the lesion was advocated. A follow-up at 3-4 weeks was done to assess the treatment response and repeat the injection if needed. Clinical photographs were taken at baseline and at every follow-up visit for the purpose of comparison.

\section{Statistical Analysis}

All the demographic and clinical data analyses were performed using SPSS version 20 (SPSS, Inc., Chicago, IL, USA). Frequencies and percentages were used for categorical variables. 

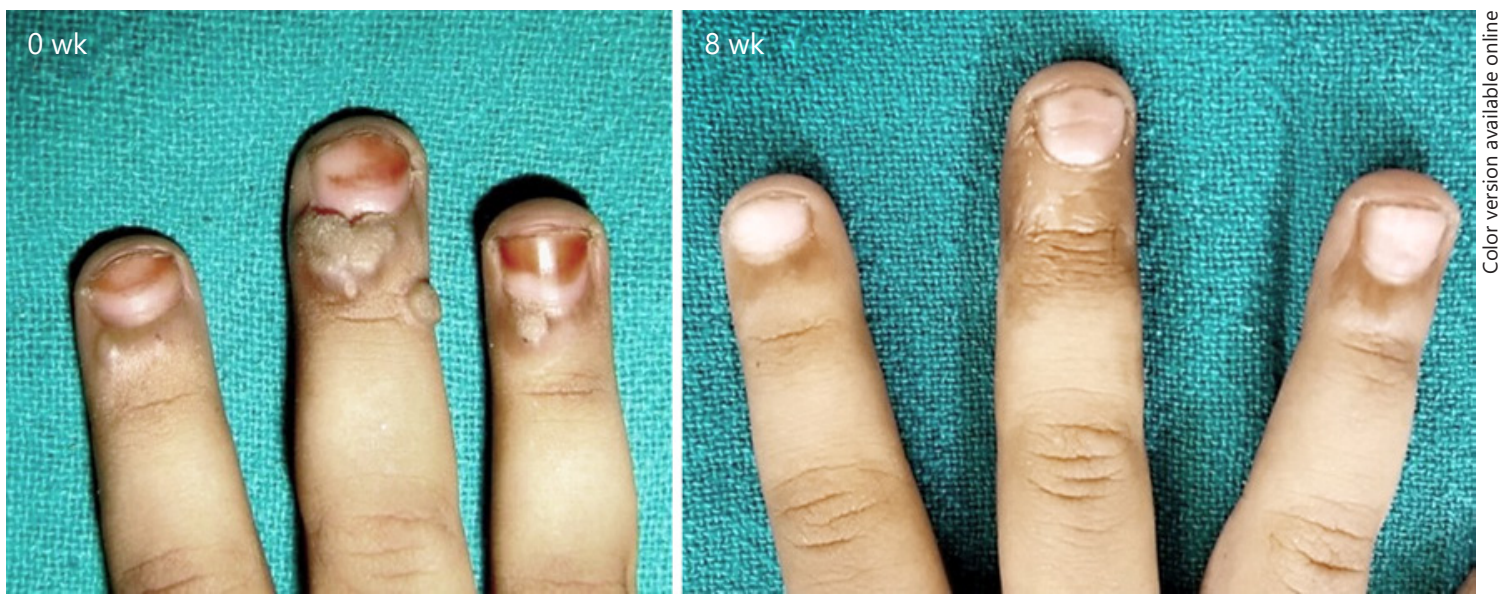

Fig. 2. a Multiple periungual warts in a young girl. b Complete resolution of warts after 2 injections with postinflammatory hyperpigmentation.

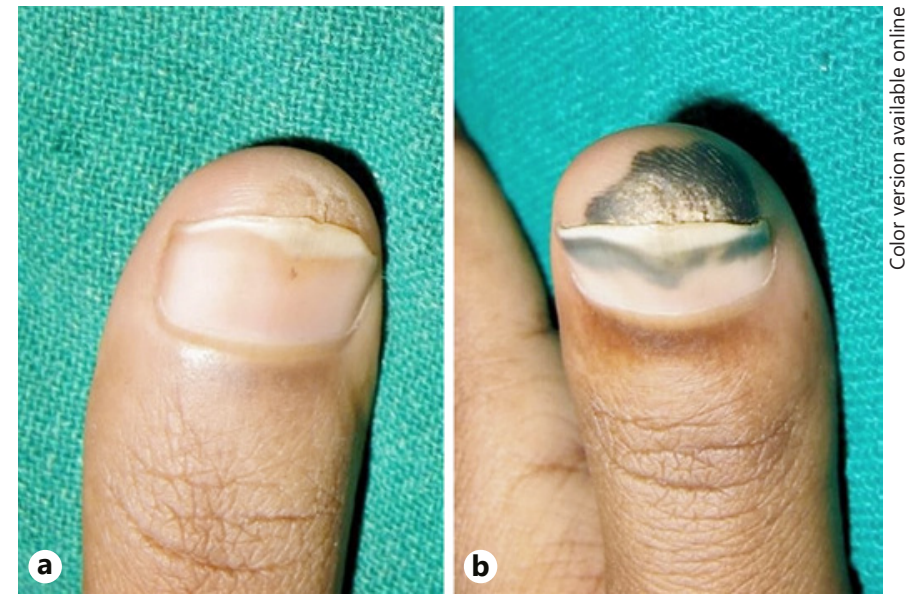

Fig. 3. a Subungual warts before treatment. b Pain and necrosis developing within $48 \mathrm{~h}$ after the 1 st injection that resolved spontaneously.

\section{Results}

\section{Demographic Profile and Clinical Characteristics}

A total of 80 patients with 250 treated warts were included in the final analysis. The mean age of the study group was 26.8 years (range 7-62 years). Males outnumbered females (52 M:28 F = 1.86:1). Duration of the warts ranged from 3 months to over 5 years (mean 8.7 months). Previous treatment was reported by $26(32.5 \%)$ patients either with no response or recurrence. Thirty-two (40\%) patients presented with a single ungual wart, while the remaining $48(60 \%)$ had multiple warts ranging from 2 to 8 in number. Of the 250 lesions, only 9 lesions (3.6\%) were present around toes, while the rest were on the hands, 187 lesions $(74.8 \%)$ were periungual, and $63(25.2 \%)$ were subungual in location. In addition, 26 (32.5\%) patients had lesions elsewhere on the body, most commonly the palms and soles, followed by the dorsa of the hands and feet, face, and forearms.

\section{Assessment and Follow-Up}

Injection bleomycin $(3 \mathrm{U} / \mathrm{mL})$ was injected at an interval of 3-4 weeks. An average of 2.61 sessions (range 2-5 sessions) per patient was required to achieve complete resolution of the warts (Fig. 1a, c). Long-standing hyperkeratotic lesions required more sessions. All patients were followed up for 3 months following resolution of lesions to look for the adverse effects and recurrence if any. Only 8 lesions $(3.2 \%)$ recurred, mostly after $8-10$ weeks. These were treated in a similar fashion.

\section{Adverse Effects}

Moderate to severe pain at the time of injection was the commonest complaint reported by $65(81.2 \%)$ patients, and it lasted for $24-48 \mathrm{~h}$ in $18(22.5 \%)$ patients. A transitory perilesional hyperpigmentation (Fig. 2a, b) was observed in 155 lesions (62\%) that resolved spontaneously in 6-8 weeks. Severe pain and necrosis (Fig. 3a, b) developed in 3 lesions $(1.2 \%)$ that was reversible without any residual scarring and deformity. No systemic side effects were noted in any patient.

\section{Discussion}

Ungual warts occur much more commonly around fingernails than around toenails; this was seen in our study as well, presumably because fingernails are more 
Table 1. Efficacy of varying strength of injection bleomycin in the treatment of periungual warts

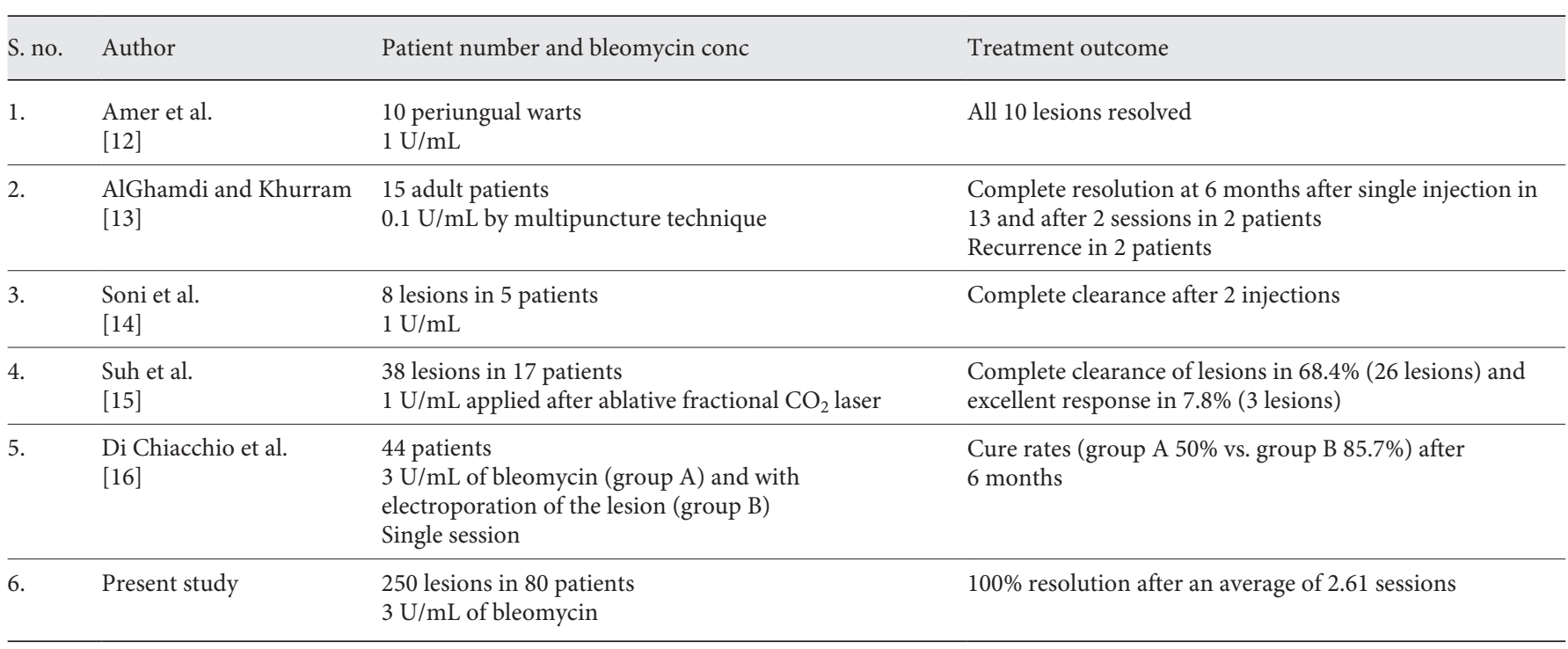

Table 2. Side effects of injection bleomycin

\begin{tabular}{ll}
\hline Possible side effects of bleomycin [refs. 17, 18] & $\begin{array}{l}\text { Side effects experienced in } \\
\text { the present study }\end{array}$ \\
\hline $\begin{array}{l}\text { Local } \\
\text { 1. Temporary nail dystrophy }\end{array}$ & $\mathrm{Nil}$ \\
2. Permanent nail dystrophy & $\mathrm{Nil}$ \\
3. Raynaud's phenomenon & $\mathrm{Nil}$ \\
4. Tissue necrosis, gangrene & $3 / 250$ lesions $(1.2 \%)$ \\
5. Loss of fingernail due to matrix destruction & $\mathrm{Nil}$ \\
6. Pain during injection & $65 / 80$ patients $(81.2 \%)$ \\
7. Transitory perilesional hyperpigmentation & $155 / 250$ lesions $(62 \%)$ \\
8. Flagellate erythema & $\mathrm{Nil}$ \\
9. Alopecia and nail changes & $\mathrm{Nil}$ \\
\hline
\end{tabular}

amenable to physical trauma during various chores and nail biting. Being on the exposed body parts, ungual warts lead to a significant cosmetic and functional handicap. Numerous treatment options are available ranging from topical therapies (10-60\% salicylic acid with or without lactic acid, imiquimod), cryotherapy, intralesional bleomycin, and immunotherapy to device-based therapies (Q-switch neodymium-doped yttrium aluminium garnet laser, pulsed dye laser, and photodynamic therapy), etc. $[5,6]$, but there is no standardized protocol. Cryotherapy may be complicated by matrix damage, surgical excision carries a high risk of recurrence, and electrosurgical ablation often leads to considerable scarring. Pulsed dye laser causes selective microvascular destruction, and therefore, periungual lesions are less responsive than palmar or common warts [6].
Bleomycin was originally isolated from the actinomycotic soil fungus Streptomyces verticillus. In addition to its antitumour properties, bleomycin exerts antibacterial and antiviral activity [7]. Intralesional bleomycin has been used as an off-label modality in the treatment of all types of warts since 1970s. This includes cutaneous, palmoplantar, and ungual warts [8-10]. In this study, we used intralesional injections of $3 \mathrm{U} / \mathrm{mL}$ bleomycin to treat 250 periungual and subungual warts.

Although there are many studies evaluating the efficacy and safety of intralesional bleomycin collectively in all types of warts (including common and palmoplantar warts), very little data are available on its efficacy in ungual warts exclusively [11]. Varying concentrations of bleomycin solution have been used in different studies due to lack of standardization. Table 1 summarizes the 
treatment results with varying strength of bleomycin solution [12-16].

The side effects reported with the use of injection bleomycin are tabulated in Table $2[17,18]$. However, with careful technique and experience, these side effects can be minimized effectively. In our study, we tried to minimize pain by prior counselling, bringing the solution to room temperature, using thin $30 \mathrm{G}$ needle, and applying ice packs to the lesion before and after the injections. No systemic side effects were noted in our study.

\section{Limitations}

The uncontrolled, open, and retrospective nature of our study is the major limitation. We plan to focus in future on controlled blinded studies using varying concentrations of bleomycin.

\section{Conclusions}

Intralesional bleomycin $(3 \mathrm{U} / \mathrm{mL})$ is a safe and effective therapeutic modality to treat periungual and subungual warts. It is associated with low recurrence and is devoid of significant side effects.

\section{Statement of Ethics}

Written informed consent was taken from all the study participants (aged 18 years and older) and from their parents or legal guardian in case of minors after explaining the study protocol and need for multiple follow-ups. The research work complies with the guidelines for human studies. The research was conducted ethically in accordance with the World Medical Association Declaration of Helsinki.

\section{Conflict of Interest Statement}

We, Dr. Archana Singal and Dr. Chander Grover, state that we have no conflicts of interest to declare.

\section{Funding Sources}

No funding was received to carry out this study.

\section{Author Contributions}

Archana Singal: concept, study design, patient recruitment, treatment and follow-up, record maintenance, literature search, and manuscript writing. Chander Grover: study design, patient recruitment, treatment and follow-up, record maintenance, and manuscript writing.

\section{References}

1 Herschthal J, McLeod MP, Zaiac M. Management of ungual warts. Dermatol Ther. 2012; 25(6):545-50.

2 Sterling JC, Handfield-Jones S, Hudson PM. Guidelines for the management of cutaneous warts. Br J Dermatol. 2001;144(1):4-11.

3 Umenzawa H, Maeda K, Takeuchi T. New antibiotics, bleomycin A and B. J Antibiot. 1966; 19:200-9.

4 Di Chiacchio NG, Di Chiacchio N, Criado PR, Brunner CHM, Suaréz MVR, Belda Junior W. Ungual warts: comparison of treatment with intralesional bleomycin and electroporation in terms of efficacy and safety. J Eur Acad Dermatol Venereol. 2019;33(12):2349-54.

5 Bacelieri R, Johnson SM. Cutaneous wart: an evidence based approach to therapy. Am Fam Physician. 2005;72:647-52.

6 Tosti A, Piraccini BM. Warts of the nail unit: surgical and nonsurgical approaches. Dermatol Surg. 2001;27(3):235-9.

7 Saitta P, Krishnamurthy K, Brown LH. Bleomycin in dermatology: a review of intralesional applications. Dermatol Surg. 2008; 34(10):1299-313.

8 Cordero AA, Guglielmi HA, Woscoff A. The common wart: intralesional treatment with bleomycin sulfate. Cutis. 1980;26(3):319-20.
9 Barkat MT, Abdel-Aziz RTA, Mohamed MS Evaluation of intralesional injection of bleomycin in the treatment of plantar warts: clinical and dermoscopic evaluation. Int J Dermatol. 2018;57(12):1533-7.

10 Shelley WB, Shelley ED. Intralesional bleomycin sulfate therapy for warts. A novel bifurcated needle puncture technique. Arch Dermatol. 1991;127(2):234-6.

11 Bik L, Sangers T, Greveling K, Prens E, Haedersdal M, van Doorn M. Efficacy and tolerability of 77 intralesional bleomycin in dermatology: a systematic review. J Am Acad Dermatol. 2020;83(3):888-903.

12 Amer M, Diab N, Ramadan A, Galal A, Salem A. Therapeutic evaluation for intralesional injection of bleomycin sulfate in 143 resistant warts. J Am Acad Dermatol. 1988;18(6): 1313-6.

13 AlGhamdi KM, Khurram H. Successful treatment of periungual warts with diluted bleomycin using translesional multipuncture technique: a pilot prospective study. Dermatol Surg. 2011;37(4):486-92.
14 Soni P, Khandelwal K, Aara N, Ghiya BC, Mehta RD, Bumb RA. Efficacy of intralesional bleomycin in palmo-plantar and periungual warts. J Cutan Aesthet Surg. 2011;4(3): 188-91.

15 Suh JH, Lee SK, Kim MS, Lee UH. Efficacy of bleomycin application on periungual warts after treatment with ablative carbon dioxide fractional laser: a pilot study [published online ahead of print, 2019 May 6]. J Dermatolog Treat. 2020 Jun:31(4):410-4.

16 Di Chiacchio NG, Di Chiacchio N, Criado PR, Brunner CHM, Suaréz MVR, Belda Junior W. Ungual warts: comparison of treatment with intralesional bleomycin and electroporation in terms of efficacy and safety. J Eur Acad Dermatol Venereol. 2019;33(12):2349-54.

17 Urbina Gonzalez F, Cristobal Gill MC, Aguilar Martinez A, Guerra Rodriguez P, Sánchez de Paz F, García-Pérez A, et al. Cutaneous toxicity of intralesional bleomycin administration in the treatment of periungual warts. Arch Dermatol. 1986;122:974-5.

18 Miller RA. Nail dystrophy following intralesional injections of bleomycin for a periungual wart. Arch Dermatol. 1984;120(7):9634 . 